\title{
Author Correction: Polycyclic aromatic chains on metals and insulating layers by repetitive [3+2] cycloadditions
}

\author{
Alexander Riss (D), Marcus Richter, Alejandro Pérez Paz (D), Xiao-Ye Wang (D), Rajesh Raju (1), Yuanqin He, \\ Jacob Ducke, Eduardo Corral, Michael Wuttke, Knud Seufert (1D, Manuela Garnica, Angel Rubio, \\ Johannes V. Barth, Akimitsu Narita (1), Klaus Müllen, Reinhard Berger, Xinliang Feng (1D, Carlos-Andres Palma \& \\ Willi Auwärter (D)
}

Correction to: Nature Communications https://doi.org/10.1038/s41467-020-15210-2, published online 20 March 2020.

The original version of this Article omitted some funding sources in the Acknowledgements. The correct version of the Acknowledgments is:

"This work was financially supported by the European Research Council Consolidator Grant NanoSurfs (no. 615233), the Advanced Grant (no. 694097), the Horizon 2020 research and innovation program 2D ink (no. 664878), and the National Science Foundation of China (no. 11974403 and Sino-German Project no. 51761135130). W.A. acknowledges funding by the DFG via a Heisenberg professorship. M.R., R.B., and X.F. thank the German Research Foundation (DFG) within the Cluster of Excellence "Center for Advancing Electronics Dresden (cfaed)" and EnhanceNano (No. 391979941). A.P.P. and A. Rubio thank the Cluster of Excellence "Advanced Imaging of Matter (AIM)" and Grupos Consolidados (IT1249-19). M.G. acknowledges funding by the H2020-MSCA-IF-2014 program under GA no. 658070 (2DNano)."

Which replaces the previous incorrect version:

"This work was financially supported by the European Research Council Consolidator Grant NanoSurfs (no. 615233), the Horizon 2020 research and innovation program 2D ink (no. 664878) and the National Science Foundation of China (no. 11974403 and SinoGerman Project no. 51761135130). W.A. acknowledges funding by the DFG via a Heisenberg professorship. M.R., R.B., and X.F. thank the German Research Foundation (DFG) within the Cluster of Excellence "Center for Advancing Electronics Dresden (cfaed)" and EnhanceNano (No. 391979941). M.G. acknowledges funding by the H2020-MSCA-IF-2014 program under GA no. 658070 (2DNano)."

This has now been corrected in both the PDF and HTML versions of the Article.

Published online: 04 August 2020

Open Access This article is licensed under a Creative Commons Attribution 4.0 International License, which permits use, sharing, adaptation, distribution and reproduction in any medium or format, as long as you give appropriate credit to the original author(s) and the source, provide a link to the Creative Commons license,
and indicate if changes were made. The images or other third party material in this article are included in the article's Creative Commons license, unless indicated otherwise in a credit line to the material. If material is not included in the article's Creative Commons license and your intended use is not permitted by statutory regulation or exceeds the permitted use, you will need to obtain permission directly from the copyright holder. To view a copy of this license, visit http://creativecommons.org/licenses/by/4.0/.

() The Author(s) 2020 